Investigating longitudinal changes in the mechanical properties of MCF-7 cells exposed to paclitaxol using particle tracking microrheology

This content has been downloaded from IOPscience. Please scroll down to see the full text. 2013 Phys. Med. Biol. 58923

(http://iopscience.iop.org/0031-9155/58/4/923)

View the table of contents for this issue, or go to the journal homepage for more

Download details:

IP Address: 141.117.7.234

This content was downloaded on 25/07/2016 at 21:14

Please note that terms and conditions apply. 


\title{
Investigating longitudinal changes in the mechanical properties of MCF-7 cells exposed to paclitaxol using particle tracking microrheology
}

\author{
Ahmed El Kaffas ${ }^{1,2,3}$, Devesh Bekah ${ }^{1,4}$, Min Rui ${ }^{1}$, J Carl Kumaradas ${ }^{1}$ \\ and Michael C Kolios ${ }^{1,2}$ \\ ${ }^{1}$ Department of Physics, Ryerson University, 350 Victoria Street, Toronto, Ontario M5B 2K3, \\ Canada \\ 2 Department of Medical Biophysics, University of Toronto, Ontario Cancer Institute, \\ Princess Margaret Hospital, 610 University Avenue, Rm 7-411, Toronto, Ontario M5G 2M9, \\ Canada \\ ${ }^{3}$ Department of Imaging Research and Physical Sciences, Sunnybrook Health Sciences Centre, \\ 2075 Bayview Ave, Toronto, Ontario M4N 3M5, Canada \\ ${ }^{4}$ Department of Biomedical Engineering, McGill University, 3775 Rue University, Montreal, \\ Quebec H3A 2B4, Canada \\ E-mail: ahmed.elkaffas@utoronto.ca
}

Received 8 July 2012, in final form 10 December 2012

Published 22 January 2013

Online at stacks.iop.org/PMB/58/923

\begin{abstract}
Evidence suggests that compression and shear wave elastography are sensitive to the mechanical property changes occuring in dying cells following chemotherapy, and can hence be used to monitor cancer treatment response. A qualitative and quantitative understanding of the mechanical changes at the cellular level would allow to better infer how these changes affect macroscopic tissue mechanical properties and therefore allow the optimization of elastographic techniques (such as shear wave elastography) for the monitoring of cancer therapy. We used intracellular particle tracking microrheology (PTM) to investigate the mechanical property changes of cells exposed to paclitaxol, a mitotic inhibitor used in cancer chemotherapy. The average elastic and viscous moduli of the cytoplasm of treated MCF7 breast cancer cells were calculated for frequency ranges between 0.2 and $100 \mathrm{rad} \mathrm{s}^{-1}$ (corresponding to 0.03 and $15.92 \mathrm{~Hz}$, respectively). A significant increase in the complex shear modulus of the cell cytoplasm was detected at $12 \mathrm{~h}$ post treatment. At $24 \mathrm{~h}$ after drug exposure, the elastic and viscous moduli increased by a total of $191.3 \mathrm{~Pa}(>8000 \times)$ and $9 \mathrm{~Pa}(\sim 9 \times)$, respectively for low frequency shear modulus measurements $\left(\right.$ at $\left.1 \mathrm{rad} \mathrm{s}^{-1}\right)$. At higher frequencies $\left(10 \mathrm{rad} \mathrm{s}^{-1}\right)$, the elastic and viscous moduli increased by $188.5 \mathrm{~Pa}(\sim 60 \times)$ and
\end{abstract}


$1.7 \mathrm{~Pa}(\sim 1.1 \times)$, respectively. Our work demonstrates that PTM can be used to measure changes in the mechanical properties of treated cells and that cell elasticity significantly increases by $24 \mathrm{~h}$ after chemotherapy exposure.

(Some figures may appear in colour only in the online journal)

\section{Introduction}

The cytoskeleton is an important determinant of the cytoplasm's mechanical properties at smaller-length scales, which in turn strongly contributes to the overall bulk mechanics of the cell at larger length-scales (Stricker et al 2010). Studies have shown that a dynamic re-organization of the cytoskeleton takes place in cells when exposed to cytotoxic agents such as chemotherapy, eventually leading to cell death (Ndozangue-Touriguine et al 2008, Wilbur et al 2007, Levee et al 1996, Suarez-Huerta et al 2000, Pelling et al 2009). Such re-organization is accompanied by changes in the cell mechanical properties, caused by cytoskeleton restructuring and nuclear condensation (Stricker et al 2010, Mammoto and Ingber 2009). These bio-mechanical changes could therefore act as treatment response markers during the course of a cancer therapy (i.e. chemotherapy).

Elastography and shear wave ultrasound are relatively new imaging modalities sensitive to the mechanical properties of tissue (Moon et al 2009, Evans et al 2010, Sebag et al 2010, Parker et al 2011). A number of clinical applications are emerging as commercial units become more readily available. Recent studies have demonstrated that these modalities can be used for the detection of tumors based on quantitative stiffness measurements (Findings et al 2012, Berg et al 2012). A number of recent studies have led us to hypothesize that both ultrasound compression and shear wave elastography imaging could also be used for cancer treatment monitoring (Bando et al 2007, Brindle 2008, English et al 2011, Hayashi et al 2012). Similarly, magnetic resonance (MR) elastography has been shown to have potential in similar clinical applications. Studies have demonstrated the use of MR elastography for diagnosis in a number of tumor models (Xydeas et al 2005, Sinkus et al 2008). More recently it has been shown to be capable of detecting treatment response in breast (Sinkus et al 2005, Yuan et al 2012, Xydeas et al 2005) and liver tumors (Venkatesh et al 2008). Furthermore, it is currently used in the clinic for monitoring liver fibrosis (Yin et al 2007). Finally, it has recently been demonstrated, in pre-clinical animal tumors, to be capable of detecting changes in the shear modulus of tumors exposed to chemotherapy (Chen et al 2011). A number of good reviews are available summarizing the use of ultrasound and MR compression and elastography/shear wave imaging in cancer applications (Sinkus et al 2008, Xydeas et al 2005). Consequently, a qualitative and quantitative understanding of how the mechanical properties of individual cells change over time after treatment administration is necessary in order to develop effective treatment monitoring tools.

In this work, we have used particle tracking microrheology (PTM) as a tool to probe the mechanical properties of individual cells treated with paclitaxol. Microrheology collectively refers to a family of techniques for measuring the mechanical properties of complex fluids, soft (bio-) materials and biological cells with relatively high temporal and spatial resolution (Waigh 2005). Active microrheology uses external forces to manipulate probe-particles and actively measure deformations, while passive microrheology uses particles driven by the thermal energy of the material to make such measurements. Passive methods have an advantage over active methods; since no large strain or stress is applied to the material, measurements remain within a linear response range without damage to the specimen under study. PTM is a passive microrheology technique, which uses the Brownian motion of embedded particles to assess 
the viscoelasticity of a material locally. Once particles are embedded in the cell (cytoplasm or nucleus), short videos of the motion of these particles are captured, from which individual particle tracks are extracted using tracking software. A mean square displacement (MSD) can then be computed for each particle and averaged over all particles. Finally, the complex shear modulus (composed of an elastic modulus $\left(\mathrm{G}^{\prime}\right)$ and a viscous modulus $\left(\mathrm{G}^{\prime \prime}\right)$ ) can be computed using a generalized version of the Stokes-Einstein relation (GSER) (Weihs et al 2006, Mason 2000).

PTM has been employed in the past to measure the mechanical properties of a variety of complex fluids, biomaterials, cells and gels (Waigh 2005). Kole et al (2004) were among the first to apply PTM as a tool to measure intracellular mechanical properties. The technique has also been used to study the differences in the mechanical properties of diseased and nondiseased cells (i.e. malignant and benign), and to investigate mechanical changes associated with cellular and subcellular processes induced by biochemical agents or mechanical forces (Dangaria and Butler 2007, Kole et al 2005, Massiera et al 2007, Panorchan et al 2006, Selvaggi et al 2010, Tseng et al 2004, 2002, Li et al 2009a, 2009b). Past investigations of the mechanical properties of cells undergoing cell death have mostly used active techniques such as atomic force microscopy (AFM). Although both passive (i.e. PTM) and active (i.e AFM) methods should in principle yield similar qualitative and quantitative measurements in live cells, this is often not the case. PTM directly probes the cytoplasm's content (at length scales of $\sim 100 \mathrm{~nm}$ ), which is often influenced by the filaments of the cytoskeleton. On the other hand, AFM tends to be more sensitive to the mechanics at larger length scales (the cell as whole). Experiments comparing PTM and AFM in malignant and benign cancer cell lines demonstrated that both techniques are sensitive to their differing mechanical properties, but that each of the techniques probed distinctive aspects of the cell mechanical properties (Li et al 2009a). To the best of our knowledge, PTM has not yet been used to measure the mechanical properties of cells exposed to chemotherapy. Our aims here are two-fold: to demonstrate as proof of principle that the PTM technique can be used to measure the average longitudinal changes in the mechanical properties of cells undergoing chemotherapy and to investigate, qualitatively and quantitatively, the mechanical property changes at different time points after drug exposure.

\section{Materials and methods}

\section{Cell culture}

MCF-7 breast cancer cells (ATCC, Manassas, VA) were cultured to 50-60\% confluence at $37{ }^{\circ} \mathrm{C}$ in $10 \%$ Dulbecco's Modified Eagle's Medium (DMEM) supplemented with $10 \%$ bovine calf serum and $10 \mu \mathrm{g} \mathrm{ml}^{-1}$ insulin. Cells were used for PTM experiments and for the cell death staining assay. For PTM experiments, cells were trypsinized and plated into four Delta-T (Bioptech, Butler, PA) culture dishes $24 \mathrm{~h}$ before microinjection (two dishes for treated and two for control). Each of the dishes was pre-marked with a $1 \mathrm{~mm}$ diameter circle, indicating the area for microinjections. For cell death staining assay, cells were trypsinized and plated into 12 culture dishes (BD Falcon, Mississauga, ON, Canada). All trypsinization occurred between passages $2-5$.

\section{Cell death staining assay}

Paclitaxol is a chemotherapeutic agent used clinically for the treatment of breast and ovarian cancer. It acts by interfering with the normal function of microtubules, hyper-stabilizing 
their structure, and eventually inducing apoptosis in most cells by $24 \mathrm{~h}$ post exposure, when administered at $20 \mathrm{ng} \mathrm{ml}^{-1}$ (Saunders et al 1997). From 12 culture dishes containing MCF-7 cells, six were treated with $20 \mathrm{ng} \mathrm{ml}^{-1}$ of paclitaxol disolved in DMSO (Bristol-Myers Squibb Canada, Montreal, QC, Canada), while six were left untreated. Cell response to paclitaxol treatment was assessed using an annexin V-FITC cell death detection kit (Sigma-Aldrich, Oakville, ON, Canada). The kit allows distinguishing between viable cells, cells in early apoptosis and cells in late apoptosis/necrosis. Cells staining with annexin V only are in the early stages of apoptosis; those staining for both annexin V and propodium iodide (PI) are in the late stage of apoptosis/necrosis, while those remaining unstained are deemed viable. Two dishes were taken out of the incubator, (one treated and one control dish) and stained at $0,6,12$ and $24 \mathrm{~h}$ after treatment. Bright field and fluorescence microscopy were used to visually confirm cell death by noting the fluorescent color to confirm both early and late cell death. Bright field and fluorescent pictures of six to eight randomly chosen FOVs for each culture dish were taken with a CCD camera at $10 \times$ magnification at each of the time-points for quantification of cell death.

\section{Particle tracking microrheology}

Cells located within the marked region (circle of $\sim 1 \mathrm{~mm}$ diameter) on the culture dish were microinjected with $0.19 \mu \mathrm{m}$ (coefficient of variation of $5 \%$ ) diameter carboxyl-modified fluorescent polystyrene microspheres (Invitrogen, Burlington, ON, Canada). After injections, cell culture dishes were placed in the incubator and left for $24 \mathrm{~h}$ in order to allow particles to diffuse through cells and for cell membrane damage repair (after the microinjection). Particles were initially dialyzed in Dulbecco's phosphate-buffered saline and prepared as described in Kole et al (2004). Each dish was taken outside the incubator for no more than 20 min for the microinjection process, after which cells were washed immediately with phosphate-buffered saline and put back into the $37^{\circ} \mathrm{C}$ incubator in DMEM culture medium, but with no serum and no insulin.

Before treatment, cell dishes were examined under the microscope and cells in which particles were successfully introduced were mapped on a sketch of the microinjection area. Two of the four dishes were treated with a $20 \mathrm{ng} \mathrm{ml}^{-1}$ concentration of paclitaxol. The other two dishes were left untreated (control). The Delta-T dishes were then sealed with a plastic transparent cover and imaged at 0,6,12 and $24 \mathrm{~h}$. Through the course of the experiments, cells were kept in an incubator and taken out only for 10-15 min imaging sessions at the specified time points. Microinjected cells were located using the sketched map within the marked area of injection in the Delta-T dishes. Videos were recorded for a total of $40 \mathrm{~s}$ at $25 \mathrm{fps}$ (yielding a total of 1000 frames per video) using a QImaging CCD camera (QImaging, Surrey, BC, Canada) attached to a PC and Streampix video capture software (Norpix, Montreal, QC, Canada). These were captured under an Olympus IX71 (Olympus, Mellville, NY) microscope at $100 \times$ magnification. Videos were kept short to avoid photobleaching of the particles and active transport of particles caused by cellular motors. A total of one to four microinjected cells were present in each video and each cell contained on average two to five particles. Approximately five to seven videos were captured for each dish at each time point for a total of 20-40 tracks per timepoint.

\section{MSD and complex shear modulus calculations}

Particles were individually tracked from the captured videos using MATLAB routines (The MathWorks, Natick, MA). These routines were originally written in IDL by Crocker (1996) 
and ported to MATLAB by Blair and Dufresne (2011). The routines were used to (1) locate particle positions for each frame, (2) filter unwanted tracks or particles (due to digitization errors, table vibrations or noticeable intracellular motor transport motion), and (3) link the positions in each frame into tracks for a collection of frames. An in-house written MATLAB GUI using the above-mentioned particle-tracking routines was used to obtain tracks from all videos. Each track was inspected individually for abrupt movement of particles (caused by table vibration/bumps). The mechanical properties of cells were computed from the particle-ensemble averaged MSD at each treatment lag-time. A minimum of at least 20 tracked particles were used to obtain the average MSD at each lag-time. All tracks were dedrifted and the MSD and complex shear modulus were computed as described by others (Blair and Dufresne 2011, Mason 2000, Kilfoil 2011, Wirtz 2009). The complex shear modulus (also known as the viscoelastic modulus) expresses the viscous modulus as the imaginary (loss) component and the elastic modulus as the real (storage) component. The use of the GSER for estimating the complex shear modulus from PTM MSD measurements in complex biomaterials and live cells was suggested by Mason (2000), and subsequently demonstrated in a number of studies (Duits et al 2009, Li et al 2009, Tseng 2002, Tseng et al 2002, Wirtz 2009). The mean of all squared displacements at a given lag-time, using all frames and particles was computed using:

$$
\left\langle\Delta r^{2}(\tau)\right\rangle=\left\langle(r(t+\tau)-r(t))^{2}\right\rangle,
$$

where $r(t)$ is a single position at a specific time point in the video, $\tau$ represents the time-scale or the lag-time at which the MSD is being computed and $\langle\ldots\rangle$ indicates time average and ensemble (over all particles) average. The viscous and elastic moduli were then extracted from the MSD as described by Mason (2000) using a simplified algebraic estimation method. In summary, the method estimates the complex shear modulus algebraically by approximating the local log-slope (log-derivative) of the MSD at each lag-time individually. This yields a smoothed approximation of the MSD, from which the slope $\alpha$ can be taken:

$$
\alpha(\tau) \equiv \frac{\mathrm{d} \ln \left\langle\Delta \overrightarrow{r^{2}}(\tau)\right\rangle}{\mathrm{d} \ln \tau} .
$$

This slope can then be used to compute the magnitude of the complex shear modulus as follows

$$
\left|G^{*}(\omega)\right|=\frac{k_{b} T}{\pi a \gamma(1+\alpha(\omega))\left\langle\Delta r^{2}\left(\frac{1}{\omega}\right)\right\rangle},
$$

where $\gamma$ represents the gamma function, $k_{b}$ is Boltzmann's constant, $a$ is the particle-probe's radius and $T$ is the temperature in Kelvins. $\left|G^{*}(\omega)\right|$ can then be computed for each lag-time (or frequency) and used to compute the elastic $\left(G^{\prime}(\omega)\right)$ modulus and viscous $\left(G^{\prime \prime}(\omega)\right)$ modulus as follows:

$$
\begin{array}{ll}
G^{\prime}(\omega) & =\left|G^{*}(\omega)\right| \cos \left(\frac{\pi \alpha(\omega)}{2}\right), \quad \text { and } \\
G^{\prime \prime}(\omega) & =\left|G^{*}(\omega)\right| \sin \left(\frac{\pi \alpha(\omega)}{2}\right) .
\end{array}
$$

The creep compliance $(\Gamma(\tau))$, which is an indication of the local deformation of cytoplasm microenvironment due to the thermally driven particles, was computed as follows:

$$
\Gamma(\tau)=\frac{3 \pi a}{2 k_{b} T}\left\langle\Delta r^{2}(\tau)\right\rangle
$$

The mechanical properties of cells were computed from the particle-ensemble averaged MSD at each treatment time-point. A minimum of at least 20 tracked particles sampled from 10-20 cells were used to obtain the MSD at each time-point. 


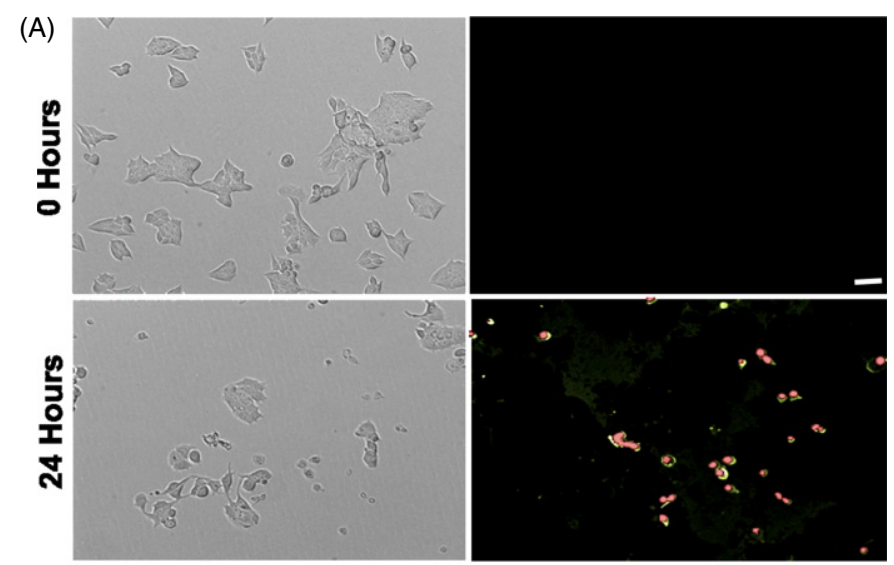

(B)

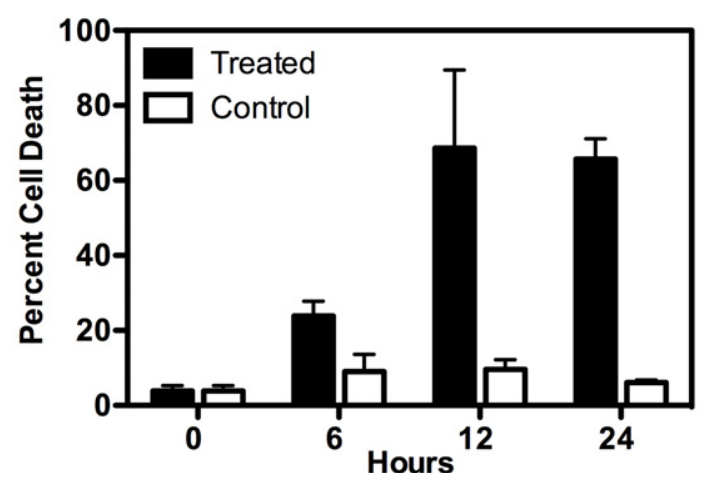

Figure 1. Cell death by paclitaxol. (A) Images from bright field microscopy (left) and fluorescent microscopy (right) of MCF-7 cells at 0 and $24 \mathrm{~h}$ after $20 \mathrm{ng} \mathrm{ml}^{-1}$ paclitaxol exposure. Images at $24 \mathrm{~h}$ indicate minimal cell colony formation after drug exposure. Cells in fluorescent images staining for annexin V (green) only indicate early apoptosis, while cells staining of a combination of annexin V (green) and PI (red) indicate late apoptosis/necrosis. By $24 \mathrm{~h}$ most cells are in either the early or late stage of apoptosis. (B) Quantification of cell death. Each bar is of six randomly chosen fields of view in a treated plate. Significant amounts of cell death (more than 65\%) are observed at 12 and $24 \mathrm{~h}$ post treatment exposure. Any observed cell death in the control population is most likely related to serum starvation. Images are taken at $10 \times$ magnification. The scale bar is $100 \mu \mathrm{m}$. Error bars represent the standard error over six field of view counts.

\section{Results}

Bright field light microscopy and fluorescent microscopy images of treated cells at 0 and $24 \mathrm{~h}$ are displayed in figure 1(A). Morphological changes of treated cells are evident in the light microscopy images at $24 \mathrm{~h}$. Corresponding images of treated cells stained with the cell death stain kit show a time dependent increase in double staining of annexin $\mathrm{V}$ (fluorescent green) and PI (fluorescent red). There are also cells at $24 \mathrm{~h}$ staining only for annexin V (fluorescent green). Cells at $0 \mathrm{~h}$ in light microscopy images appeared mostly viable and display well-formed cell colonies. Minimal cell death staining was visible in control dishes (not shown). Figure 1(B) displays the quantified ratio of non-viable or dying cells in fluorescent images (staining for both early and late stage cell death) to all cells counted in corresponding bright field images. These results indicate a time-dependent increase in cell death and is 
(A)

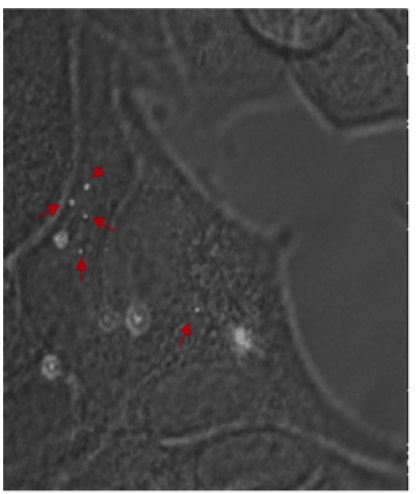

(B)

\section{Hours}

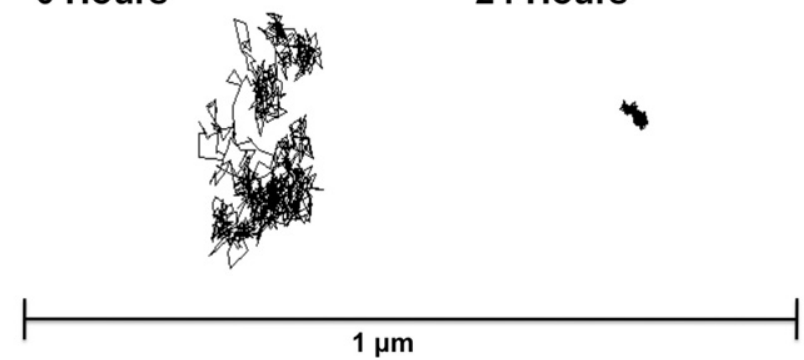

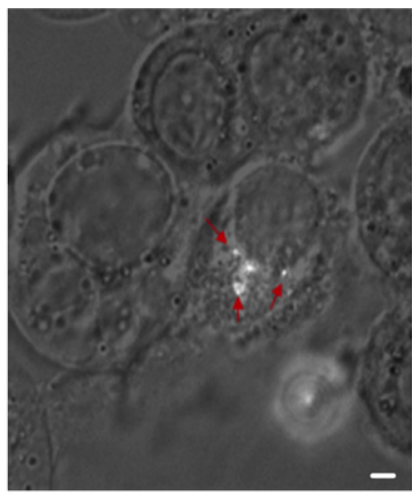

24 Hours

Figure 2. (A) Representative images of particles embedded in a single MCF-7 cell at 0 and $24 \mathrm{~h}$. A rounded cell undergoing morphology associated with cell death is shown. Arrows indicate well resolved particles that are tracked. The scale bar is $1 \mu \mathrm{m}$. (B) Representative $30 \mathrm{~s}$ tracks of two different particles injected in MCF-7 cells at 0 and $24 \mathrm{~h}$ after exposure to paclitaxol. At the start of treatment, beads travel greater distances compared to $24 \mathrm{~h}$ after drug exposure, indicating stiffening of cytoplasm.

consistent with other paclitaxol dose-studies on MCF-7 cells (Saunders et al 1997). By $12 \mathrm{~h}$, more than $65 \%$ of cells were dead or dying, while remaining cells appeared morphologically different from control cells.

Figure 2(A) is a representative image of particles embedded in the cytoplasm of a viable cell at 0 and $24 \mathrm{~h}$ post treatment with paclitaxol. Figure 2(B) highlights representative tracks of a single particle at 0 and $24 \mathrm{~h}$ after treatment. In figure 3(A), the average MSD (left $Y$-axis) and the creep compliance (equation (6); right $Y$-axis) is plotted against lag-time for treated and non-treated cells, at each time point. A time-depedent decrease in cytoplasm deformability was observed in the creep compliance. Figure 3(B) illustrates the MSD of each particle used to obtain the average MSD at each of the time points. For treated cells, the number of tracks at each time point was: $0 \mathrm{~h} n=26$ tracks, $6 \mathrm{~h} n=38$ tracks, $12 \mathrm{~h} n=20$ tracks, $24 \mathrm{~h} n=21$ tracks. For control cells, the number of tracks at each time point was: $0 \mathrm{~h} n=26$ tracks, $6 \mathrm{~h}$ $n=38$ tracks, $12 \mathrm{~h} n=20$ tracks, $24 \mathrm{~h} n=21$ tracks.

We tested the average MSD of particles for statistical significance between 0 and $6 \mathrm{~h}$, 0 and $12 \mathrm{~h}$, and 0 and $24 \mathrm{~h}$ after paclitaxol exposure for two differing lag-times $(0.1$ and $1 \mathrm{~s}$ ) in treated and untreated cells. These were chosen as to represent particle MSDs at the shorter and longer lag-times. The difference in the MSD in treated and untreated cells was evaluated using a two-sample t-test (two-tailed; assuming unequal variances; $\alpha=0.05$ ). At 


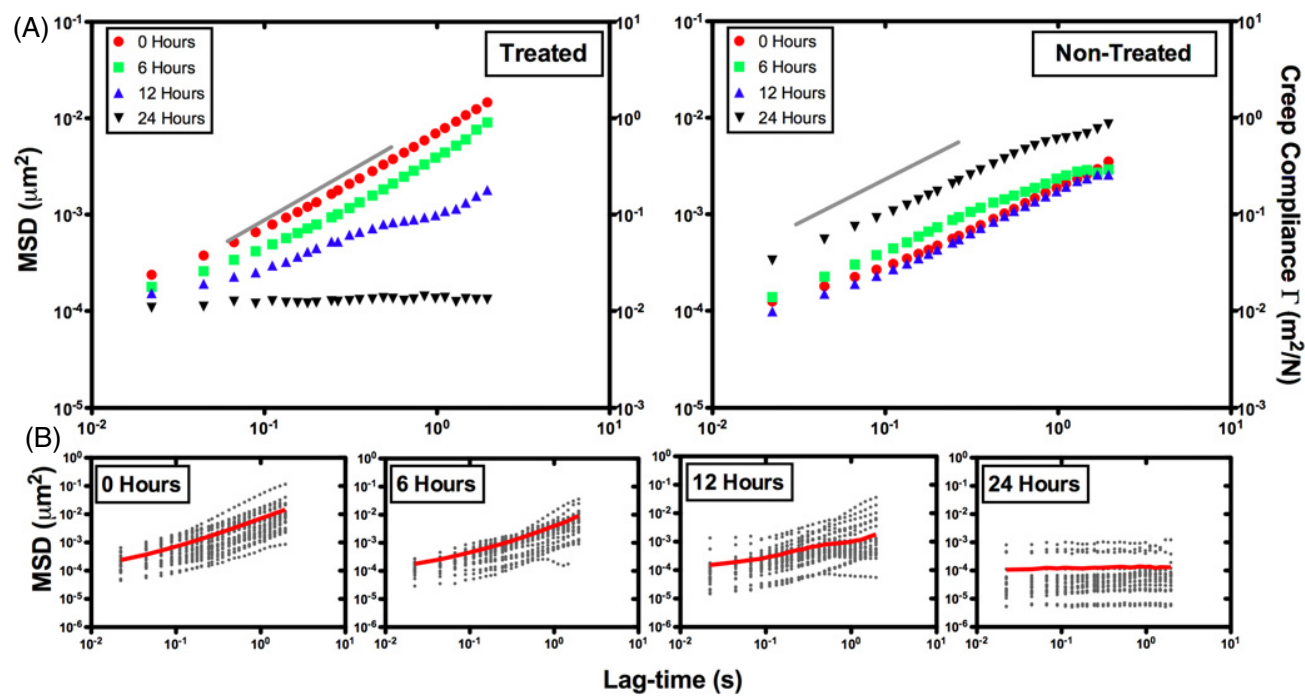

Figure 3. (A) Average MSD of particles as a function of lag-time in treated and control cells at $0,6,12$ and $24 \mathrm{~h}$. A decrease in the MSD slope is observed as the treatment time increases. The creep compliance scale is shown on the right $Y$-axis. The MSD change of log-slope is characteristic of an increasing real and imaginary parts of the complex shear modulus in the particle-probe's microenvironment. The log-slope change is noticeable starting at $12 \mathrm{~h}$. At $24 \mathrm{~h}$, the MSD has a log-slope of $\sim 0$, indicating a predominantly elastic cytoplasm. No statistically significant change in the MSD magnitude nor the slope of control cells was found. A slope of 1 in the log-log MSD graphs indicates that the cell cytoplasm is viscoelastic, while a slope greater than 1 or smaller than 1 indicates a predominantly viscous or elastic cell cytoplasm, respectively. Our findings suggest that the intracellular microenvironment remains consistent over $24 \mathrm{~h}$ in viable cells. Changes in MSD amplitude are most likely related to cytoskeleton filament re-organization during cell mitosis or motility. Gray solid line indicates log-slope of 1. Creep compliance of treated cells, which describes the local deformation of the cytoplasm microenvironment by the thermally driven microinjected particles is directly proportional to the MSD. Data indicates a time-dependent decrease in local deformability of the cytoplasm of paclitaxol treated cells. (B) All MSDs for all tracks measured in treated cells at each time point. $(0 \mathrm{~h}$ with $n=26$ tracks; $6 \mathrm{~h}$ with $n=38$ tracks; $12 \mathrm{~h}$ with $n=$ 20 tracks; 24 h with $n=21$ tracks). The distribution of particle MSDs became wider through the course of the treatment. MSD slope changes were apparent at $12 \mathrm{~h}$ in some of the tracks. By $24 \mathrm{~h}$, all tracked MSDs had a slope of $\sim 0$. The MSD was found to be significantly different between 0 and $12 \mathrm{~h}$ as well as 0 and $24 \mathrm{~h}$ at a $1 \mathrm{~s}$ lag-time $(p=0.05)$.

Table 1. Summary of $p$-values for statistical tests.

\begin{tabular}{lllll}
\hline & Treated $(0.1 \mathrm{~s})$ & Control $(0.1 \mathrm{~s})$ & Treated $(1 \mathrm{~s})$ & Control $(1 \mathrm{~s})$ \\
\hline 0 and $6 \mathrm{~h}$ & $* 0.04$ & 0.50 & 0.15 & 0.53 \\
0 and $12 \mathrm{~h}$ & 0.38 & 0.30 & $* 0.05$ & 0.32 \\
0 and $24 \mathrm{~h}$ & $* p<0.0001$ & 0.70 & $* p<0.0001$ & 0.40 \\
\hline
\end{tabular}

the $0.1 \mathrm{~s}$ lag-time, the MSD decreased between the 0 and $6 \mathrm{~h}$, and the 0 and $24 \mathrm{~h}$ time points $(p \leqslant 0.05)$. At the $1 \mathrm{~s}$ lag-time, the MSD decreased between the 0 and 12 , and the 0 and $24 \mathrm{~h}$ time points $(p \leqslant 0.05)$. No other statistical differences were found for treated and untreated conditions at these lag-times. Table 1 has a summary of all $p$-values.

The complex shear modulus was computed (see equation (5)) for treated cells at 0,6 , 12 and $24 \mathrm{~h}$ after drug administration and plotted on a log-log graph against frequency (figures 4(A) and (B)). The elastic and viscous moduli were plotted for the 0 and $24 \mathrm{~h}$ time 

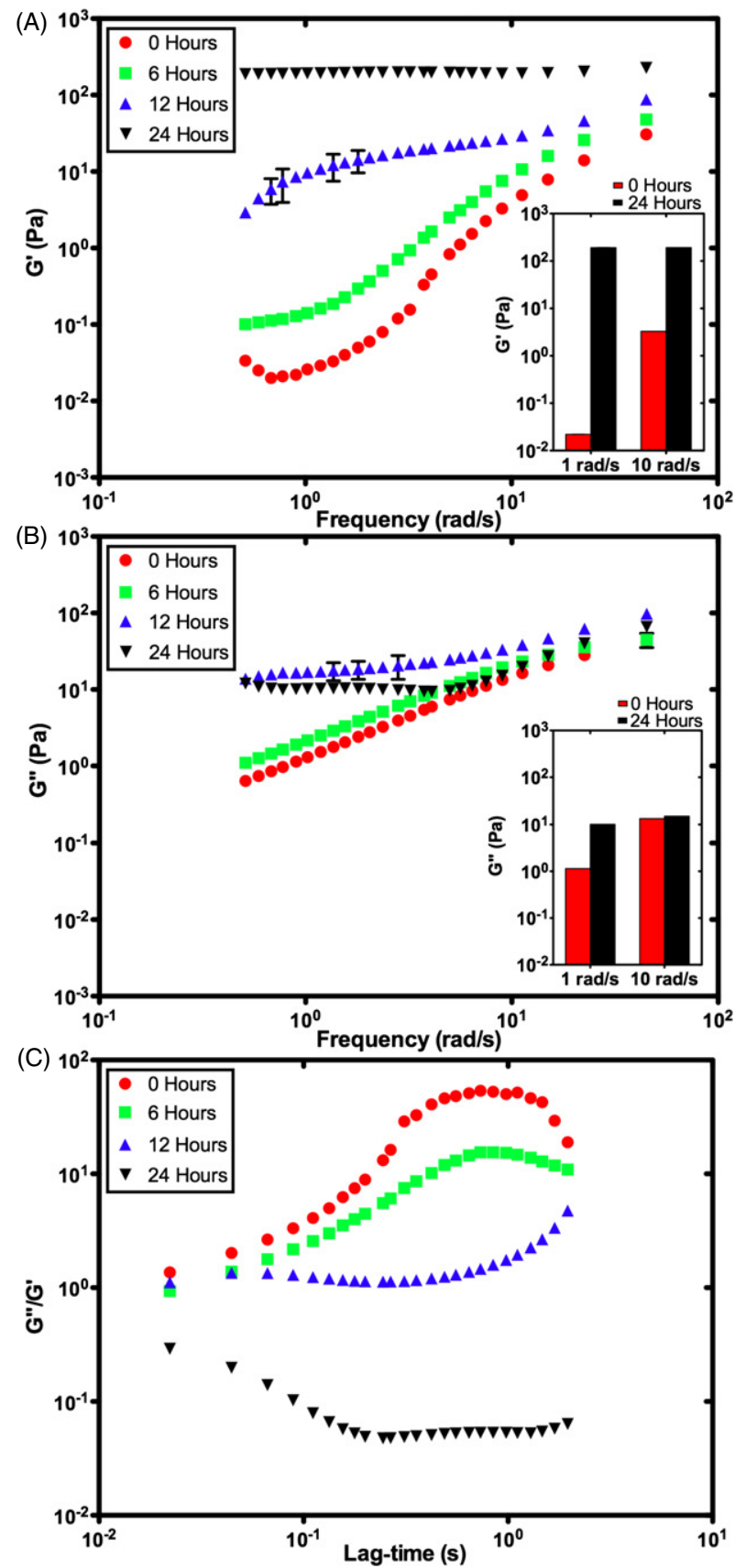

Figure 4. (A) Elastic $\left(\mathrm{G}^{\prime}\right)$ and $(\mathrm{B})$ viscous $\left(\mathrm{G}^{\prime \prime}\right)$ moduli as a function of probing frequency for treated cells at four drug exposure times. An increase in both moduli is observed at lower frequencies over the treatment time course. No change in the viscous modulus is measured at the higher frequencies. Embeded bar graphs in (A) and (B) are of the same measurment $\left(\mathrm{G}^{\prime}\right.$ or $\mathrm{G}^{\prime \prime}$ respectively) but shown at $1 \mathrm{rad} \mathrm{s}^{-1}$ and $10 \mathrm{rad} \mathrm{s}^{-1}$. Error bars for standard errors are shown for some of the time point means ( $0 \mathrm{~h} n=26$ tracks; $6 \mathrm{~h} n=38$ tracks; $12 \mathrm{~h} n=20$ tracks; $24 \mathrm{~h} n=21$ tracks). (C) Ratio of $\mathrm{G}^{\prime \prime} / \mathrm{G}^{\prime}$ for treated cells, which indicates the relative contributions of the viscous and elastic component of the cell at different time points after therapy. 
points in sub-figures (figures 4(A) and (B)) in order to demonstrate the increase in cytoplasm elasticity. This was done for the 1 and $10 \mathrm{rad} \mathrm{s}^{-1}$ frequencies as to avoid edge truncation errors when taking the MSD slope (Mason 2000). At $24 \mathrm{~h}$ after drug exposure, the elastic and viscous moduli increased from 0.022 to 191.3 and from 1.1 to $10.1 \mathrm{~Pa}$, respectively (at $1 \mathrm{rad} \mathrm{s}^{-1}$ ). At higher frequencies (at $10 \mathrm{rad} \mathrm{s}^{-1}$ ), the elastic and viscous moduli increased from 3.3 to $191.8 \mathrm{~Pa}$ and from 13.4 to $15.1 \mathrm{~Pa}$, respectively. Figure $4(\mathrm{C})$ is of the $\mathrm{G}^{\prime \prime} / \mathrm{G}^{\prime}$ ratio, which is indicatory of the relative contribution of the elasic and viscous component as a function of lag-time, relative to one-another, within the intracellular microenvironment.

\section{Discussion}

In this study, we have demonstrated that PTM can successfully detect changes in the mechanical properties of breast cancer cells in a longitudinal chemotherapy study. Furthermore, we show that on average, the complex shear modulus measured in the cytoplasm of paclitaxol treated cells experiences a time-dependent increase over a 24 h time-course. More than $60 \%$ of cells treated with paclitaxol underwent cell death by $24 \mathrm{~h}$ after drug exposure (figure 1), consistent with other studies (Saunders et al 1997). Cells that did not undergo cell death by $24 \mathrm{~h}$ were likely in the early phase of treatment response, exhibiting minor morphological changes or microtubule destabilization. Such effects would have also contributed to the mechanical property changes of cells measured in this work. Untreated cells remained viable (figure 1(B)), with the exception of a small percentage of cells.

The average MSD at each of the treatment time-points in figure 3(A) is representative of all tracked particles located throughout each microinjected cell's cytoplasm (figure 3(B)). Hence, the mechanical property measurements are representative of all microinjected cells that remain adhered to the dish at the time of video capture; this average is representative of cells responding to treatment as well as those potentially not responding to the treatment. Representative tracks shown in figure 2(B) indicate that the MSD decreased after treatment and that the average cytoplasmic environment is stiffening. Similar cytoplasmic stiffening has been reported in other studies (Lam et al 2007, Levee et al 1996, Pelling et al 2009).

The significant decrease in the average MSD amplitude of treated cells at 6 (significant at $0.1 \mathrm{~s}$ lag time), 12 (significant at $1 \mathrm{~s} \mathrm{lag-time)} \mathrm{and} 24 \mathrm{~h}$ (significant at both the 0.1 and $1 \mathrm{~s}$ lag-times) indicates an increase in both the real and imaginary parts of the complex shear modulus during cell death (figure 4). The MSD of treated cells significantly changed $24 \mathrm{~h}$ post treatment at both lag-times tested. However, statistical significance of the MSD amplitude was only apparent for the $0.1 \mathrm{~s}$ lag-time at the $6 \mathrm{~h}$ time point and for the $1 \mathrm{~s} \mathrm{lag-time} \mathrm{at} \mathrm{the} 12 \mathrm{~h}$ time point. The trend observed at the shorter lag-time $(0.1 \mathrm{~s})$ suggests that the cells may undergo periods of stiffening, followed by periods of relaxation during cell death. A similar stiffening and relaxation trend has previously been reported (Pelling et al 2009, Levee et al 1996). On the other hand, at the $1 \mathrm{~s}$ lag-time, changes in the average MSD amplitude only became significant $12 \mathrm{~h}$ post drug exposure. We posit that no significant change was observed at the $1 \mathrm{~s}$ lag-time of the $6 \mathrm{~h}$ treatment time-point MSD due to variations in individual particle MSD log-slope. This is likely associated with variations in individual cell response time. Mechanical changes not directly linked with cell death are likely related to paclitaxol effects on the microtubule of cells (Saunders et al 1997).

Changes in the MSD log slope were observed by $12 \mathrm{~h}$ post treatment (figure 3 ). A slope of 1 in the log-log MSD graphs indicates that the cell cytoplasm is viscoelastic, while a slope greater than 1 or smaller than 1 indicates a predominantly viscous or elastic cell cytoplasm, respectively. We observed an MSD log slope approaching 0 for particles tracked at 12 and $24 \mathrm{~h}$ after treatment, suggesting that the particle-probe microenvironment becomes more 
elastic. This is likely associated with cytoskeleton re-organization (polymerization) during cell death (Levee et al 1996, Mammoto and Ingber 2009, Ndozangue-Touriguine et al 2008, Suarez-Huerta et al 2000).

For untreated (control) cells, the changes in MSD as a function of time are small (figure 3). No changes in the average MSD slope are observed, while some variations in the average MSD amplitude of individual particles are measured. However, the average MSD log-slope did not change significantly from one time point to another in control conditions, which further supports the notion that changes in the PTM results are representative of mechanical changes related to paclitaxol exposure. MSD amplitude variations in control cells are likely linked to cell mechanical property heterogeneity. For example, Tseng et al (2002) showed, using PTM, that beads located closer to the nucleus experience a stiffer cellular microenvironment than those located at the edges of the cytoplasm of a cell. Variations in the MSD over time could also be associated with changes in cell cycle, mitosis or cell motility.

PTM measurements are also susceptible to artifacts caused by other cell activities. Intracellular molecular motors cause directed motion, cell mobility/crawling can cause spurious tracer motion, while reaction forces from trafficking cytoskeleton tread-milling or remodeling can further 'jiggle' the mesh network surrounding the tracked particle. However, it is suggested that such non-Brownian motion usually dominates at longer lag-times, while Brownian motion usually dominates at shorter lag-times (Wirtz 2009, Crocker and Hoffman 2007). In this study, we have taken precautions to minimize non-Brownian fluctuations. These precautions include: (i) microinjecting particles in order to avoid particle engulfment by intracellular motors, (ii) using carboxyl-modified fluorescent polystyrene particles to decrease the chances of intracellular active motor binding, (iii) taking short particle motion videos in order to minimize active cellular motor-driven motion (focusing on shorter lag-times), and (iv) averaging a large number of particles from multiple cells to obtain the average MSD (Li et al 2009a, 2009b, Duits et al 2009, Wirtz 2009, Kole et al 2004, Tseng et al 2002, Valentine et al 2004). It is also possible that thermal energy production varies between viable and dying cells. A decrease or increase in the heat production would then lead to MSD amplitude changes of thermally driven motion that are not directly related to the mechanical properties of dying cells. However, we anticipate that the slope would remain independent of frequency, and would directly characterize the particle microenvironment.

We have computed the complex shear modulus by using the GSER (Mason 2000). As treatment time progressed, both the elastic $\left(\mathrm{G}^{\prime}\right)$ and viscous $\left(\mathrm{G}^{\prime \prime}\right)$ moduli increased (figures $4(\mathrm{~A})$ and $(\mathrm{B})$ ) while the creep compliance $(\Gamma(\tau))$ decreased (figure $3(\mathrm{~A})$ ). Our results indicate that the measured complex shear modulus undergoes the most significant changes at lower frequencies $\left(0.03-0.3 \mathrm{rad} \mathrm{s}^{-1}\right)$. By $24 \mathrm{~h}$, the elastic modulus remained relatively constant over the entire range of frequencies. This is indicative that the cytoplasm of cells becomes more solid like $24 \mathrm{~h}$ after paclitaxol exposure. The changes in the complex shear modulus suggest that paclitaxol causes the cell cytoplasm to become more elastic and viscous over time. However, we note from figure $4(C)$ that the $G^{\prime \prime} / G^{\prime}$ ratio changes as a function of treatment time. The $\mathrm{G}^{\prime \prime} / \mathrm{G}^{\prime}$ ratio specifies whether the probed microenvironment is predominantly viscous or elastic; a ratio greater than one indicates that the viscous component is dominant, while a ratio smaller than one indicates that the elastic component is dominant. We found that at the 0 and $6 \mathrm{~h}$ time points, the $\mathrm{G}^{\prime \prime} / \mathrm{G}^{\prime}$ ratio was strongly viscous at the lower probing frequencies and more elastic at the higher probing frequencies. On the other hand, at the $12 \mathrm{~h}$ time point, the $\mathrm{G}^{\prime \prime} / \mathrm{G}^{\prime}$ ratio remained almost constant and equal to one over most of the frequency range, with the exception of the lower frequencies. This suggests that the intracellular microenvironment has a strong elastic component by this time point. Finaly, we found that by $24 \mathrm{~h}$ after therapy, the $G^{\prime \prime} / G^{\prime}$ ratio falls below one, inidcating a predominantly elastic microenvironment. These 
results suggest that the elastic and viscous contributions to the intracellular microenvironment change as a function of both probing frequency and treatment time. It is important to note that the GSER models the cytoplasm of cells as a complex fluid, and is based on three main assumptions: that the probe is embedded in a completely homogeneous material, that it has 'no-slip' boundary conditions and that its fluctuations are thermally driven (Mason 2000). There are studies though that suggest that at least one of these assumptions may not hold true in cells (Crocker et al 2000, Valentine et al 2004), causing single particle tracking MSD results to report only a correct frequency dependence, with incorrect amplitude measurements in cells (Crocker and Hoffman 2007, Wirtz 2009). However, by taking this one step further and averaging over a number of particles distributed spatially across the cell's cytoplasm, and over multiple cells, estimates of the mechanical properties are then more likely to represent absolute values. Furthermore, recent studies by Li et al (2009a), (2009b) and Duits et al (2009) suggest that the intracellular location of the particle may be relatively unimportant when an ensemble of particles are used to obtain an average MSD, and that measurements from microinjected particles are more likely to report measurements dictated by the actin cytoskeleton than the microtubule network.

Our results demonstrate that the cytoplasm of in vitro treated cells becomes stiffer as these respond to treatment. Such mechanical changes could potentially be exploited with compression or shear wave elastography imaging to monitor cancer treatment response in diseased tissue. Spatial variations of the shear modulus are generally orders of magnitude greater than variations of the longitudinal modulus (dominant in conventional clinical ultrasound) in soft tissues (Moon et al 2009, Garra 2007). Because of this, significant soft tissue contrast can be observed when imaging with such modalities. Elastography and shear wave ultrasound imaging have been used in clinical trials for the differentiation of benign and malignant breast tumors with considerable success (Tanter et al 2008, Evans et al 2010, Sebag et al 2010). Experiments are now underway to measure the changes in shear wave propagation as a function of treatment, while evidence that elastography can detect such changes have been reported (Bando et al 2007, Brindle 2008, English et al 2011, Hayashi et al 2012).

Future work will include detailed studies of the longitudinal response of individually treated cells in a more controlled environment. An experimental set-up that would allow us to do so would yield better insight on the dynamics of the changes in mechanical properties during chemotherapy. In these experiments, it will be essential to consider how the mechanical property changes vary at different spatial locations within the cell, and which part of the cell (cytoplasm vs. nucleus vs. membrane) is most closely linked to the whole cell's mechanical properties during treatment response (Tseng et al 2002). Finally, the use of mathematical tools (i.e. finite element models) could help model and better understand the response of individual cells and ensembles of cells exposed to treatment, and measured using compression and shear wave elastography, at different stages of therapy. This would in turn allow to effectively optimize treatment monitoring imaging modalities, such as the ones described here. We have begun developing such models for individual and collections of cells; we anticipate that our experimental PTM results can be used as input parameters for such models, leading to a better understanding of tumor response to chemotherapy.

\section{Conclusion}

We have shown that intracellular PTM can be used to study the mechanical property changes of cells undergoing chemotherapy. It was observed that the MSD of microinjected particles became much smaller with time, as apoptosis was induced in MCF-7 cells. The MSD as a function of lag-time was shown to decrease both in magnitude and slope over the treatment 
time course, which suggests that treatment causes cells to become more elastic and viscous over time. At $24 \mathrm{~h}$ after treatment, a $60 \times$ and a $>8000 \times$ increase in the elastic modulus was observed at the 10 and $1 \mathrm{rad} \mathrm{s}^{-1}$ frequencies, respectively. A $1.1 \times$ increase and a $9 \times$ increase was observed for the viscous modulus for frequencies of 10 and $1 \mathrm{rad} \mathrm{s}^{-1}$, respectively. This is the first time that PTM has been shown to estimate changes in local mechanical properties of dying cells from measurments of intracellular particle displacements.

\section{Acknowledgments}

The authors thank Arthur Worthington, Candice Schreiber and Hamed Basseri for technical help with the experimental set up as well as Dr Gregory J Czarnota for valuable scientific advice. The authors would like to acknowledge funding from the NSERC discovery grant program (grant 216986-07). This research was undertaken, in part, thanks to funding from the Canada Research Chairs Program awarded to Dr M Kolios. Funding to purchase the equipment was provided by the Canada Foundation for Innovation, the Ontario Ministry of Research and Innovation, and Ryerson University.

\section{References}

Bando $\mathrm{H}$ et al 2007 Real-time ultrasound elastography for monitoring tumor response to neoadjuvant chemotherapy in primary breast cancer J. Clin. Oncol. 2514023

Berg W A et al 2012 Shear-wave elastography improves the specificity of breast US: the BE1 multinational study of 939 masses Radiology 262 435-49

Blair D and Dufresne E 2011 Matlab particle tracking http://physics.georgetown.edu/matlab/ (last accessed on May 2, 2011)

Brindle K 2008 New approaches for imaging tumour responses to treatment Nature Rev. Cancer 8 94-107

Chen J et al 2011 Assessment of early tumor response to chemotherapy using MR elastography (MRE) Proc. 19th Annu. Meeting Int. Soc. Mag. Ros. Med. (Montreal, 7-13 May 2011) p 3112

Crocker J 1996 Methods of digital video microscopy for colloidal studies J. Colloid Interface Sci. 179 298-310

Crocker J C and Hoffman B D 2007 Multiple-particle tracking and two-point microrheology in cells Methods Cell Biol. 83 141-78

Crocker J C, Valentine M T, Weeks E R, Gisler T, Kaplan P D, Yodh A G and Weitz D A 2000 Two-point microrheology of inhomogeneous soft materials Phys. Rev. Lett. 85 888-91

Dangaria J H and Butler P J 2007 Macrorheology and adaptive microrheology of endothelial cells subjected to fluid shear stress Am. J. Physiol. Cell Physiol. 293 C1568-75

Duits M et al 2009 Mapping of spatiotemporal heterogeneous particle dynamics in living cells Phys. Rev. E 79 1-11

English R et al 2011 Use of ultrasound elasticity imaging to monitor the response of a primary breast cancer to neoadjuvant chemotherapy in one patient in a pilot study Breast Cancer Res. 13 P43

Evans A et al 2010 Quantitative shear wave ultrasound elastography: initial experience in solid breast masses Breast Cancer Res. 12 R104

Findings E et al 2012 Invasive breast cancer: relationship between shear-wave elastographis findings and histologic prognostic factors Radiology $263673-7$

Garra B S 2007 Imaging and estimation of tissue elasticity by ultrasound Ultrasound Q. 23 255-68

Hayashi M et al 2012 Evaluation of tumor stiffness by elastography is predictive for pathologic complete response to neoadjuvant chemotherapy in patients with breast cancer Ann. Surg. Oncol. 19 3042-9

Kilfoil M 2011 Biological physics laboratory for cell mechanics http://people.umass.edu/kilfoil/downloads.html (last accessed on May 2, 2011)

Kole T P, Tseng Y and Wirtz D 2004 Intracellular microrheology as a tool for the measurement of the local mechanical properties of live cells Methods Cell Biol. 78 45-64

Kole T P et al 2005 Intracellular mechanics of migrating fibroblasts Mol. Biol. Cell 16 328-38

Lam W A, Rosenbluth M J and Fletcher D A 2007 Chemotherapy exposure increases leukemia cell stiffness Blood 109 3505-8

Levee M G et al 1996 Actin polymerization and depolymerization during apoptosis in HL-60 cells Am. J. Physiol. 271 C1981-C1992

Li Y, Schnekenburger J and Duits M 2009a Intracellular particle tracking as a tool for tumor cell characterization J. Biomed. Opt. 14064005 
Li Y, Vanapalli S A and Duits M H G 2009b Dynamics of ballistically injected latex particles in living human endothelial cells Biorheology 46 309-21

Mammoto A and Ingber DE 2009 Cytoskeletal control of growth and cell fate switching Curr. Opin. Cell Biol. $21864-70$

Mason T G 2000 Estimating the viscoelastic moduli of complex fluids using the generalized Stokes-Einstein equation Rheol. Acta 39 371-8

Massiera G et al 2007 Mechanics of single cells: rheology, time dependence, and fluctuations Biophys. J. 93 $3703-13$

Moon W K et al 2009 Analysis of elastographic and B-mode features at sonoelastography for breast tumor classification Ultrasound Med. Biol. 35 1794-802

Ndozangue-Touriguine O, Hamelin J and Bréard J 2008 Cytoskeleton and apoptosis Biochem. Pharmacol. 76 11-8

Panorchan P et al 2006 Microrheology and ROCK signaling of human endothelial cells embedded in a 3D matrix Biophys. J. 91 3499-507

Parker K J, Doyley M M and Rubens D J 2011 Imaging the elastic properties of tissue: the 20 year perspective Phys. Med. Biol. 56513

Pelling A E et al 2009 Mechanical dynamics of single cells during early apoptosis Cell Motil. Cytoskeleton 66 409-22

Saunders D E et al 1997 Paclitaxel-induced apoptosis in MCF-7 breast-cancer cells Int. J. Cancer 70 214-20

Sebag F et al 2010 Shear wave elastography: a new ultrasound imaging mode for the differential diagnosis of benign and malignant thyroid nodules J. Clin. Endocrinol. Metab. 95 5281-8

Selvaggi L et al 2010 Multiple-particle-tracking to investigate viscoelastic properties in living cells Methods 51 20-6

Sinkus R et al 2005 Viscoelastic shear properties of in vivo breast lesions measured by MR elastography Magn. Reson. Imaging 23 159-65

Sinkus R et al 2008 Potential of MRI and ultrasound radiation force in elastography: applications to diagnosis and therapy Proc. IEEE 96 490-9

Stricker J, Falzone T and Gardel M L 2010 Mechanics of the F-actin cytoskeleton J. Biomech. 43 9-14

Suarez-Huerta N et al 2000 Actin depolymerization and polymerization are required during apoptosis in endothelial cells J. Cell. Physiol. 184 239-45

Tanter M et al 2008 Quantitative assessment of breast lesion viscoelasticity: initial clinical results using supersonic shear imaging Ultrasound Med. Biol. 34 1373-86

Tseng Y 2002 Local dynamics and viscoelastic properties of cell biological systems Curr. Opin. Colloid Interface Sci. 7 210-7

Tseng Y, Kole T P and Wirtz D 2002 Micromechanical mapping of live cells by multiple-particle-tracking microrheology Biophys. J. 83 3162-76

Tseng Y et al 2004 Micro-organization and visco-elasticity of the interphase nucleus revealed by particle nanotracking J. Cell Sci. 117 2159-67

Valentine M T, Perlman Z E, Gardel M L, Shin J H, Matsudaira P, Mitchison T J and Weitz D A 2004 Colloid surface chemistry critically affects multiple particle tracking measurements of biomaterials Biophys. J. 86 4004-14

Venkatesh S K et al 2008 MR elastography of liver tumors: preliminary results Am. J. Roentgenol. 190 1534-40

Waigh T A 2005 Microrheology of complex fluids Rep. Prog. Phys. 68 685-742

Weihs D, Mason T G and Teitell M 2006 Bio-microrheology: a frontier in microrheology Biophys. J. 91 4296-305

Wilbur L A, Fletcher M J and Rosenbluth D A 2007 Chemotherapy exposure increases leukemia cell stiffness Blood $1093505-8$

Wirtz D 2009 Particle-tracking microrheology of living cells: principles and applications Annu. Rev. Biophys. 38 301-26

Xydeas T et al 2005 Magnetic resonance elastography of the breast Invest. Radiol. $40412-20$

Yin M et al 2007 Assessment of hepatic fibrosis with magnetic resonance elastography Clin. Gastroenterol. Hepatol. 5 1207-13.e2

Yuan H, Guzina B B and Sinkus R 2012 Application of topological sensitivity toward tissue elasticity imaging using magnetic resonance data J. Eng. Mech. (available online, doi:10.1061/(ASCE)EM.1943-7889.0000472) 